Revista de Psicología Vol. 31 (1), 2013 (ISSN 0254-9247)

\title{
Efecto de una plataforma virtual en comprensión de lectura y vocabulario: Una alternativa para mejorar las capacidades lectoras en primaria*
}

\author{
Cecilia Thorne $(\dagger)^{1}$, Kim Morla ${ }^{2}$, Paola Uccelli ${ }^{3}$, Teresa Nakano ${ }^{4}$, \\ Beatriz Mauchi ${ }^{5}$, Lorena Landeo ${ }^{6}$, Angie Vásquez ${ }^{7}$ y Román Huerta ${ }^{8}$ \\ Pontificia Universidad Católica del Perú
}

\begin{abstract}
Frente a los bajos resultados que los niños peruanos obtienen en las pruebas nacionales e internacionales de comprensión de lectura y tomando en consideración el aumento de inversión en tecnología en las instituciones educativas del país, el presente estudio buscó desarrollar y evaluar una herramienta virtual para mejorar la comprensión de lectura. Para ello, se adaptaron las estrategias de comprensión de lectura y ejercicios de vocabulario del entorno virtual ICON y se diseńó la plataforma LEO. Un total de 88 estudiantes de quinto grado de primaria, provenientes de colegios privados de nivel socioeconómico medio-bajo de Lima Metropolitana, participaron en este estudio cuasi-experimental, con un grupo control y un grupo que participó en la intervención digital a lo largo de 12 semanas. Todos los participantes fueron evaluados utilizando pruebas de entrada y salida de comprensión de lectura y vocabulario. Los resultados revelaron que los estudiantes que interactuaron con la plataforma LEO obtuvieron resultados significativamente más altos en la comprensión de textos narrativos y vocabulario luego de finalizada la intervención, respecto a sus puntajes al inicio de la misma y también en comparación con el grupo que no empleó la plataforma. Palabras clave: comprensión de lectura, TIC, plataforma virtual, estrategias de comprensión de lectura, vocabulario.
\end{abstract}

Effects of a virtual platform in reading comprehension and vocabulary: An alternative to improve reading abilities in Elementary school

Given, on the one hand, the poor results obtained by Peruvian children in the national and international reading assessments. And on the other hand, the increased investment in technology for schools in the country, this study aimed to develop and test an online tool to improve reading comprehension. In order to do this, the reading comprehension strategies and vocabulary activities from the research-based digital environment ICON were adapted to design the platform LEO. A total of 88 fifth graders from urban middle-to-low-income private schools from Lima participated in this quasi-experimental study, which involved a control group and a treatment group that participated in a 12-week teacher-mediated digital intervention. All participants were administered reading and vocabulary assessments pre 
and post intervention. Results revealed that students who participated in the intervention achieved higher comprehension scores for narrative texts and higher vocabulary scores than those of the control group.

Keywords: reading comprehension, ICT, virtual platform, reading comprehension strategies, vocabulary.

* El entorno virtual ICON (Improving Comprehension Online) es un proyecto desarrollado por el Centro de Aplicación de Tecnologías Especiales - CAST (Center for Applied Special Technologies) junto con equipos de investigación de Boston College, Harvard University y Vanderbilt University. Tiene por objetivo mejorar la comprensión lectora de estudiantes de quinto de primaria, incluyendo aquellos cuya primera lengua no es el inglés, por medio del trabajo de ejercicios de vocabulario y estrategias de comprensión de lectura (Proctor, Dalton, Uccelli \& Snow, 2008).

1 Doctora en Psicología de la Katholiek Universiteit Nijmegen. Fue profesora principal del Departamento de Psicología de la PUCP, miembro fundador del Colegio de Psicólogos del Perú y directora de la Revista de Psicología de la PUCP.

2 Magíster en Automatización y Computadoras del Instituto Politécnico de Bucarest Gheorghe Gheorghiu-Dej. Fue docente de Ingeniería Informática a nivel de pregrado y posgrado de la PUCP. Directora adjunta de la Dirección de Informática Académica de la PUCP. Contacto: kmorla@pucp.edu.pe

3 Doctora en Educación de la Universidad de Harvard. Profesora asociada de la Escuela de Postgrado en Educación de la Universidad de Harvard. Contacto: paola_uccelli@gse.harvard.edu

4 Magíster en Políticas Públicas y Sociales de la Universidad Pompeu Fabra. Docente del Departamento de Psicología de la PUCP. Coordinadora de Proyectos de Investigación de la Dirección de Informática Académica de la PUCP. Dirección postal: Av. Universitaria 1801, San Miguel, Lima-Perú. Contacto: tnakano@pucp.edu.pe.

5 Doctora en Educación de la Universidad Católica del Perú. Profesora principal del Departamento de Humanidades de la PUCP. Contacto: bmauchi@pucp.edu.pe

6 Magíster en Educación de la Universidad de Harvard. Consultora senior de la Dirección Nacional de Información y Evaluación de la Calidad Educativa del Ministerio de Educación de la Nación Argentina. Consultora del Centro de Estudios en Políticas Públicas de Argentina. Contacto: lorena.landeo@gmail.com

7 Bachiller en Psicología de la Pontificia Universidad Católica del Perú. Asistente de docencia en el Departamento de Psicología de la PUCP. Asistente de investigación de la Dirección de Informática Académica de la PUCP. Contacto: angie.vasquez@pucp.edu.pe

8 Bachiller en Ingeniería Informática de la Pontificia Universidad Católica del Perú. Asistente de docencia en el Departamento de Informática de la PUCP. Asistente de Desarrollo Informático de la Dirección de Informática Académica de la PUCP. Contacto: rhuerta@pucp.edu.pe 
Leer es una capacidad fundamental para la vida cotidiana y el aprendizaje. Leemos el mundo desde que nacemos, pues interpretamos información del medio para darle significado (Condemarín, Galdames \& Medina, 2002 citados en Claux \& La Rosa, 2004). Desde pequeños, estamos expuestos a distintos estímulos que requieren nuestra lectura (Chall, 1996; Whitehurts \& Lonigan, 1998), y esta también es necesaria para acceder a las diversas tecnologías actuales y a los diferentes campos de conocimiento (Strickland, 2002; Thorne, 1991). Incluso, se ha encontrado que mientras más alto es el nivel de comprensión de lectura, mayor es el desarrollo estructural de las naciones (La Tercera, 2000 citado en Pizarro, Muńoz, Colarte, Hernández \& Olmos, 2005). Dada su importancia, la lectura es un tema ampliamente investigado. Actualmente, se le reconoce como una actividad constructiva que involucra una serie de procesos cognitivos y psicolingüísticos complejos (Braslavsky, 2005; Bravo, Villalón \& Orellano, 2005), principalmente la decodificación y la comprensión. Por decodificación se entiende la capacidad para descifrar y pronunciar correctamente las palabras impresas. Si bien la decodificación automática y fluida es necesaria, esta habilidad está muy lejos de ser suficiente para garantizar la comprensión de un texto. La investigación reciente define la comprensión de lectura como el proceso que consiste en simultáneamente extraer y construir significados al interactuar con un texto escrito (Snow, 2002). Esta definición enfatiza la importancia de ambos aspectos del proceso: extracción (decodificación) y construcción (comprensión). Ciertamente, extraer la información del texto es esencial, pero la comprensión será posible solo mediante la integración de esta información con los conocimientos previos y el contexto sociocultural específicos del lector.

El desenvolvimiento de este proceso supone la interacción entre lector y el texto (Gómez-Palacio, Gonzáles \& López-Arraiza, 1993). Es decir, el lector interpreta y reconstruye lo que lee a partir de sus propias 
experiencias, conocimientos, valores y creencias (Durkin, 1978; Goodman, 1982; Tinker \& McCullough, 1962 citados en Braslavsky, 2005). Por su parte, sobre el tema Snow (2002) propone tres elementos: (a) el lector, (b) el texto, y (c) la actividad. El primero contempla las capacidades, la motivación, la personalidad, el estado físico, las experiencias y los conocimientos de la persona que lee; además, considera un aspecto de desarrollo asociado al nivel sociocognitivo del lector. El segundo, el texto, puede ser de muy diversos tipos (narrativo, descriptivo, argumentativo), constar de estructuras lingüísticas más o menos complejas (vocabulario, sintaxis, conectores) y puede presentarse en distintos formatos (impreso o digital). El tercer elemento es la actividad que incluye el porqué y para qué se lee, es decir, el propósito y las consecuencias de la lectura (nuevos aprendizajes y experiencias). Así pues, no es lo mismo leer un libro de texto para preparase para un examen de ciencia que leer una novela por placer en la playa, o revisar los avisos del periódico en busca de un puesto de trabajo. La lectura en cada una de estas situaciones está motivada por propósitos muy distintos, que llevan incluso al mismo lector a tener estándares de comprensión muy diferentes y a aplicar estrategias de comprensión distintas (Perfetti, Landi \& Oakhill, 2005).

En tal sentido, un lector necesita integrar una multitud de conocimientos y habilidades que responden al tipo de texto leído y a la actividad realizada. Por un lado, para leer un texto narrativo, un lector necesita destrezas lingüísticas para entender las cadenas referenciales, las estructuras sintácticas y los significados de palabras recurrentes en este tipo de textos (Snow \& Uccelli, 2009); requiere destrezas sociocognitivas para atribuir sentimientos y estados mentales que expliquen las acciones de los personajes (Selman, 2003); y también demanda saberes previos sobre el mundo, pues todo texto asume cierto conocimiento sin el cual es imposible comprender lo leído (Snow, 2002). Asimismo, la comprensión de una narración se guía por la consideración de las estructuras globales de este tipo de textos (introducción, clímax y resolución) (Perfetti et al., 2005). Por otro lado, un texto expositivo (por ejemplo, de ciencias naturales o sociales) presenta retos 
Efecto de una plataforma virtual en comprensión de lectura y vocabulario / Thorne et al.

distintos, puesto que su organización global, las características del lenguaje empleado y el tipo de conocimientos previos que necesitan ser activados son diferentes. Por supuesto, todo proceso de comprensión está además altamente influenciado por la motivación del lector, aspecto crucial que actualmente constituye un particular desafío con la población adolescente (Alverman \& Eakle, 2003; Guthrie, Wigfield \& Perencevich, 2004; Moje \& Hinchman, 2004).

En el ámbito educativo es necesario precisar con mayor detalle qué se entiende por una comprensión exitosa en la interacción de los estudiantes con un texto escrito. En este caso, el término "comprensión" es vago y puede usarse para referirse a varios niveles de logros en la lectura. Para precisarlos, resulta especialmente útil la distinción entre recordar lo leído y aprender de lo leído (Kintsch \& Kintsch, 2005). Si la decodificación de un texto constituye solo un paso inicial para descifrar la información que contiene, la comprensión superficial constituye solo el simple proceso de recordarla. En efecto, la repetición textual o la respuesta correcta a una pregunta literal no indican una real comprensión de lo leído, pues recordar no es comprender. Más bien, la comprensión, según los modelos cognitivos más recientes, se alcanza al integrar la información textual con los conocimientos previos para lograr un cambio en las representaciones mentales del lector, es decir, al construir lo que los autores anteriormente mencionados llaman modelo situacional. En esta misma línea, Pérez (2005) plantea cinco niveles de comprensión según su complejidad: comprensión literal, reorganización de la información, comprensión inferencial, lectura crítica o valorativa, y apreciación lectora.

Todo ello describe la complejidad del proceso de la lectura y el reto al que deben hacer frente los educadores: generar espacios y actividades didácticas que engranen los múltiples aspectos involucrados para formar lectores exitosos. La instrucción constituye, sin duda, uno de los mediadores que ejerce mayor influencia en el éxito de este proceso (Cazden \& Beck, 2005; Snow, 2002), especialmente si se considera que la lectura constituye una habilidad escolar fundamental para acceder a las demás disciplinas escolares (Strickland, 2002; Thorne, 1991). 
Sin embargo, los resultados de las evaluaciones nacionales e internacionales señalan serias deficiencias del sistema escolar para lograr las capacidades esperadas en la comprensión de lectura. La Evaluación Censal de Estudiantes 2011 (Ministerio de Educación, 2012) reporta que el $29.8 \%$ de estudiantes de segundo de primaria alcanza el nivel esperado para su grado en comprensión de textos (es decir, ser capaz de deducir el tema central de un texto, establecer relaciones causa-efecto explícitas e identificar datos explícitos). Aunque se observa un progreso a lo largo del tiempo (16\% en el $2007,16.9 \%$ en el $2008,23.1 \%$ en el 2009 , y $28.7 \%$ en el 2010), todavía más de la mitad del alumnado no alcanza estas capacidades (Ministerio de Educación, 2008a, 2010a, 2011).

A nivel internacional, los resultados educativos de Perú se ubican por debajo de la media del resto de países de la región (Ministerio de Educación, 2001, 2008b, 2010b). Aunque se ha obtenido un incremento, los resultados del Programa Internacional de Evaluación de Estudiantes - PISA 2009 posicionan a Perú como el tercer país más bajo de los 65 participantes a nivel mundial y el último en comparación con ocho de la región. Además, cerca del $80 \%$ de estudiantes no llegan al tercer nivel en comprensión de lectura de un total de seis establecidos (Ministerio de Educación, 2010b). Al respecto, las investigaciones realizadas en las dos últimas décadas confirman los hallazgos de las evaluaciones comentadas anteriormente. Carreño (2000), Morales (1999), Nakano (1996), Pinzás (1993), Thorne (1991), y Thorne y Nakano (2001) dan cuenta del pobre desarrollo de las habilidades de decodificación y comprensión lectora de los niños peruanos desde el inicio de su escolaridad. Más aún, en un estudio llevado a cabo en Lima, Thorne y Pinzás (1988) encontraron que muchos maestros de primaria se inclinan a tomar la lectura como sinónimo de decodificación. Estos resultados sugieren que existe una errónea concepción sobre el proceso de desarrollo de la comprensión de lectura. Así, se considera que esta aparece naturalmente cuando los niños logran descifrar un texto, por lo que no se enseñan estrategias de comprensión (resumir, preguntar, imaginar pasajes de la lectura, reflexionar, predecir, entre otras), cuyo uso constituye una forma efectiva para mejorar la comprensión lectora 
de los estudiantes (Dalton \& Proctor, 2007; Pressley, 2002; Stickland, 2002; Williams, 2007).

De igual manera, el vocabulario ha sido identificado como uno de los principales predictores de la comprensión lectora en varias lenguas (Morales, 2009; Stahl \& Nagy, 2006; Verhoeven \& Perfetti, 2011). Se ha llegado a estimar que un lector que no puede entender el $90 \%$ de las palabras de un texto, no podrá comprender lo que lee y, por lo tanto, no podrá tampoco aprender las palabras desconocidas únicamente a través de la lectura independiente. Por ello, es necesaria una intervención explícita que permita expandir el vocabulario de los lectores para que puedan contar con las herramientas necesarias para comprender un texto (Morales, 2009; Proctor, Uccelli, Dalton \& Snow, 2009; Stahl \& Nagy, 2006). Como consecuencia de esta situación, surge la necesidad de capacitar a los docentes en la enseñanza de la comprensión lectora (Chesterfield, Culver, Hunt \& Linan-Thompson, 2005; Morales, 2009; Villalón, Silva, Razmilic \& Swartz, 2005), más aún cuando los estudios revelan que muchas veces los maestros no están debidamente preparados para enseñar a leer y a comprender (Pressley, 2002).

En resumen, la realidad descrita muestra tres aspectos importantes a ser analizados: (a) la inequidad existente en el país, pues los bajos resultados se acentúan en las escuelas públicas, especialmente en las zonas rurales y más pobres del país (Ministerio de Educación, 2001, 2008a, 2010a); (b) los estudiantes con bajo rendimiento en el inicio de la lectura continúan con dificultades a lo largo de su escolaridad, mientras que aquellos con adecuada preparación y buen inicio en la lectura continúan siendo buenos lectores (Bravo et al., 2005; Pizarro et al., 2005); y, (c) gran cantidad de profesores no cuentan con las capacidades requeridas para enseñar y desarrollar la comprensión de lectura en sus alumnos, pues consideran relevante únicamente el aprendizaje de la decodificación. Bajo este panorama, es de vital importancia encontrar alternativas eficientes que permitan a las escuelas mejorar la capacidad de comprensión de lectura de los estudiantes. En este sentido, la incorporación de las tecnologías de la información y las comunicaciones (TIC) aparece como una 
alternativa para optimizar los procesos de enseñanza-aprendizaje. Es decir, facilitaría al sistema escolar apropiarse de nuevas metodologías y maneras diferentes de organizar la gestión educativa (Pedró, 2011). Así, lograr esta sinergia entre las TIC y el proceso educativo supone planificar estratégicamente el uso de las herramientas. Tal como señala Pedró (2011): “(...) un fácil acceso es condición necesaria, pero no suficiente, para que la tecnología en la escuela responda a las expectativas existentes" (p. 19). Una muestra de ello son los hallazgos de Villatoro y Silva (2005) sobre las dificultades de los maestros para incorporar las TIC en su práctica pedagógica; por ello, se concibe a la capacitación docente como elemento central del éxito de los programas educativos con TIC (Sunkel, 2006; Trinidad, 2005; Villatoro \& Silva, 2005).

En el Perú, al igual que en otros países de la región, se ha planteado incorporar las TIC como parte de un plan integral para la mejora de la calidad educativa (Villatoro \& Silva, 2005), la reducción de la brecha digital y el fomento de la equidad (Sunkel, 2006). A partir del año 2000, el Ministerio de Educación implementó el Plan Huascarán con el objetivo de proporcionar recursos tecnológicos e informáticos y acceso a Internet a los centros educativos. De igual modo, desde el 2007, el piloto Una laptop por niño pretendió beneficiar a los estudiantes de las zonas rurales. No obstante, en un estudio llevado a cabo en el departamento de Ayacucho, Trinidad (2005) reporta que los maestros generalmente señalaron como único objetivo del programa la dotación de computadoras, sin incluir el aspecto pedagógico. Ello muestra una seria dificultad para incorporar estas herramientas en los procesos de enseñanza-aprendizaje.

Dadas estas dificultades, resulta comprensible que las experiencias que integren las TIC para el desarrollo de la lectura aún sean escasas en Latinoamérica, si bien se encuentran algunos esfuerzos en países como México y Chile (Ponce, López, Labra, Brugerolles \& Tirado, 2007; Sunkel, 2006). Los entornos web, como ICON o Raz-Kids, ofrecen alternativas a los docentes para desarrollar y mejorar la comprensión de lectura. Sin embargo, a pesar de que actualmente existe poca información sobre los logros o avances de este tipo de programas 
en el fortalecimiento de la comprensión lectora, la mayoría de iniciativas reconoce que las concepciones teóricas y pedagógicas sobre este tema pueden enriquecerse explorando y experimentando materiales que no implican necesariamente los recursos impresos (Henao, 2006). Así, tal como lo manifiestan Henao y Ramírez (2007): “el análisis de las estrategias que entran en juego durante la lectura y la comprensión de textos en formato [virtual e] hipermedial está contribuyendo a cualificar e innovar los modelos y las prácticas de enseñanza-aprendizaje de la lecto-escritura" (p. 48). Por lo tanto, la realidad descrita requiere encontrar e implementar estrategias que innoven el proceso de enseñanza-aprendizaje de la comprensión de textos.

El presente estudio plantea una modalidad que incorpora las TIC, pues busca aprovechar los laboratorios que se encuentran implementados en los colegios y se basa en el desarrollo de una metodología de aprendizaje efectiva que considera el vocabulario y las estrategias de comprensión de lectura. Asimismo, contempla la evaluación del proceso de modo que, en un futuro, pueda ser adaptada y empleada por docentes de diversos grados y de zonas menos favorecidas. Para ello, se utilizó como punto de partida la plataforma digital bilingüe (español e inglés) desarrollada sobre la base de investigaciones recientes y diseñada en el marco del proyecto ICON (Dalton, Proctor, Uccelli, Mo \& Snow, 2011; Proctor et al., 2009). Este programa consta de un entorno virtual que incluye textos narrativos e informativos acompañados de soportes pedagógicos para estimular la comprensión lectora, y también incluye actividades diseñadas para expandir el vocabulario y estimular el uso de estrategias de comprensión de lectura. De acuerdo con lo anterior, se llevó a cabo un estudio sobre el funcionamiento y el efecto del entono virtual. Se planteó como objetivo general evaluar la eficacia del programa LEO para mejorar la comprensión de lectura y el aprendizaje de vocabulario de estudiantes de quinto grado de primaria. Es decir, al finalizar la intervención, se esperó que los estudiantes que emplearon la plataforma obtuvieran mejores resultados que aquellos que no lo hicieron. Asimismo, de manera complementaria, se analizó el desempeño de los estudiantes en las actividades del programa y la experiencia de las docentes que lo llevaron a cabo. 


\section{Método}

En la presenta investigación se empleó un diseño cuasi-experimental con asignación aleatoria de grupos (aula experimental y aula control).

\section{Participantes}

Los participantes fueron dos docentes y 88 alumnos de quinto de primaria, procedentes de dos instituciones educativas privadas de nivel socioeconómico medio bajo de Lima Metropolitana. Los colegios participantes forman parte de la red de instituciones educativas con las que colabora la Dirección de Informática Académica (DIA) de la Pontificia Universidad Católica del Perú, y fueron seleccionados mediante un muestreo intencional. Dos criterios de selección se tomaron en cuenta: (1) que el colegio contara con un laboratorio de cómputo multimedia equipado con una computadora por alumno y con acceso a Internet, y (2) que la docente del aula asignada al grupo experimental aplicara el programa dentro del horario de clase de Comunicación. Las dos docentes participantes reportaron haber completado sus estudios de docencia universitarios. Una de ellas señaló poseer 34 años de experiencia en educación primaria, mientras que la otra 11 ańos en dicho nivel y 17 como docente en total.

Las niñas y los niños participantes presentaron una edad promedio de 10.3 años. Se dividieron en dos grupos: el grupo experimental, integrado por 49 estudiantes ( 21 varones y 28 mujeres) que utilizaron el Programa LEO; y el grupo control, conformado por 39 estudiantes (11 varones y 28 mujeres). La asignación de los participantes a cada grupo se realizó por colegio y al azar a nivel de aula, con lo que se tuvieron dos aulas para el grupo experimental y dos para el control.

\section{Medidas e instrumentos de medida}

Plataforma LEO. El entorno virtual se desarrolló a partir del modelo de la plataforma ICON, diseñada para favorecer el aprendizaje autónomo, teniendo en cuenta los diversos estilos de aprendizaje de 
los estudiantes (Proctor et al., 2008). Por ello, entre sus características principales se encuentran la interacción constante y la retroalimentación inmediata, así como el empleo de diferentes estímulos sensoriales (audio, imagen, texto y video), tipos de ejercicios y formas de respuesta (opción múltiple, respuesta abierta, relacionar dos alternativas, seleccionar imágenes o palabras, entre otros). La plataforma LEO incluye ocho textos, cuatro narrativos y cuatro informativos, cuya extensión varía entre cuatro y diez páginas. Los textos narrativos están basados en leyendas de diversos lugares del mundo y los textos informativos presentan datos variados sobre el tema principal que se desprenden de los narrativos. Cada lectura se apoya en imágenes, audio y palabras subrayadas que forman parte de un glosario de la lectura. Además, LEO acompaña cada texto con tareas de vocabulario, estrategias de comprensión de lectura y actividades de evaluación:

- Tareas de vocabulario: proponen dos tipos de ejercicios, uno de exploración del significado de la palabra (Explorar) y otro de profundización de los significados recién adquiridos (Excavar). También incluye la tarea "Agregar a mi glosario" que permite al estudiante construir su propio léxico con palabras de su elección, cuyo significado desconoce o atrae por algún otro motivo.

- Estrategias de comprensión de lectura: incluyen seis estrategias de comprensión lectora, las cuales han sido identificadas como efectivas en lectores exitosos (resumir, preguntar, predecir, imaginar, sentir y clarificar). Para cada una de ellas se diseñó un ejercicio que debe ser resuelto al finalizar la lectura de cada página del texto, de manera que el lector tiene que detener su lectura en ciertos puntos específicos para interactuar activamente con el texto mediante el uso de una estrategia.

- Actividades de evaluación: consisten en un examen de vocabulario y uno de comprensión de lectura. En el primero se evalúa el uso de las nuevas palabras aprendidas mediante diferentes tipos de pregunta y se organizan las respuestas en tres niveles de dificultad: Hábil (baja), Experto (media) y Sabio (alta). En el segundo se mide la comprensión de lectura del estudiante a nivel literal e inferencial. 
El estudiante solo puede acceder a desarrollar las actividades de evaluación una vez que ha culminado con las tareas de vocabulario y los ejercicios sobre las estrategias de comprensión de lectura.

En la figura 1 se resumen las actividades que trabaja el alumno en la plataforma LEO.

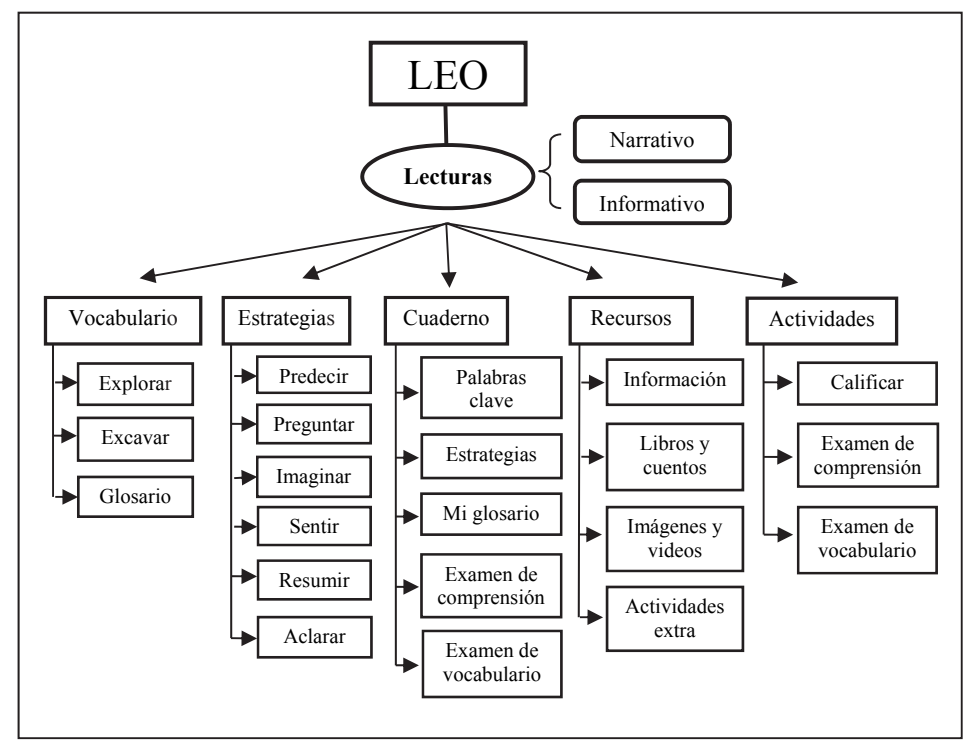

Figura 1. Organización de la plataforma LEO desde la vista del alumno

Adicionalmente, el entorno cuenta con pestañas donde los estudiantes pueden revisar todas sus respuestas (Cuaderno) y encontrar links a webs, videos y diversa información que complementa la lectura (Recursos). También, es posible verificar los resultados de sus evaluaciones comparándose con el promedio de su aula y consultar los mensajes de retroalimentación de su docente. Para los profesores, la plataforma ofrece además un espacio para monitorear el trabajo de sus estudiantes y enviarles mensajes con comentarios sobre su desempeńo. Finalmente, contiene un módulo que les permite a los maestros crear textos con sus propios ejercicios, recursos, vocabulario y evaluaciones. 
Adaptación del PIRLS Reading Literacy Test. La exploración de las habilidades relacionadas con la comprensión de lectura se realizó según los resultados de la adaptación del PIRLS Reading Literacy Test —empleado en la evaluación internacional PIRLS llevada a cabo por la International Association for the Evaluation of Educacional Achievement en el año 2000, realizada por Morales (2009), para evaluar niños de cuarto y quinto de primaria de nivel socioeconómico medio y bajo de Lima. La adaptación explora cuatro habilidades relacionadas con la comprensión de lectura: extraer información, realizar inferencias, interpretar e integrar información y analizar los contenidos del texto. La prueba se compone de 22 preguntas, algunas abiertas y otras de opción múltiple: 11 corresponden a un texto narrativo y las 11 restantes a uno informativo. Para el primero se obtuvo un coeficiente de confiabilidad de .76 y para el segundo de .72 .

Evaluación de vocabulario. La medición de la adquisición del vocabulario trabajado en la plataforma se realizó con un instrumento construido para fines del presente estudio. Las palabras se seleccionaron a partir de una muestra al azar del total de palabras que conforman el glosario del programa. Consiste en 25 ítems con un formato de respuesta de opción múltiple organizados según los cinco tipos de ejercicios desarrollados por LEO: completar oraciones, relacionar una palabra con una imagen, identificar sinónimos, identificar antónimos y responder preguntas sobre una oración que contiene una palabra clave. El análisis de confiabilidad por consistencia interna presenta un coeficiente de .70 con el método de covariación de ítems y .78 con el método de división por mitades. Además, se estableció la validez de contenido al asegurarse que los ítems correspondían a palabras del programa y a los tipos de ejercicios que este desarrolla.

Guia de observación de aula. La información sobre el desarrollo de las sesiones de aplicación se recogió a través de la Guía de observación de aula, consistente en 11 preguntas (seis abiertas y cinco cerradas) que se llenan cada 15 minutos durante seis intervalos. Se registran las actividades de la plataforma efectuadas, los textos resueltos, la participación de los alumnos, la guía y retroalimentación del docente, además de las dificultades que se presentaron. 
Guias de preguntas para las reuniones con las docentes. Tuvieron por objetivo sistematizar la experiencia de los profesores al aplicar la plataforma. Se prepararon tres formatos: la Guía para la reunión individual, la Guía de reunión docente grupal y el Cuestionario de reflexión. La primera se conformó de cuatro preguntas orientadas a recoger sus opiniones sobre los beneficios y dificultades percibidos con el programa. La segunda constó de diez preguntas que, aparte de lo anterior, incluyen elementos específicos de la plataforma y aprendizajes de las docentes. Finalmente, el tercero agrega preguntas sobre el acompañamiento brindado y cómo integrar mejor el trabajo de la plataforma con los contenidos de clase.

\section{Procedimiento}

Adaptación y desarrollo de la plataforma LEO. Esta etapa consideró dos aspectos importantes: la adaptación de los contenidos y la adecuación tecnológica. La adaptación de los contenidos ofrecidos por ICON se centró en mejorar y adecuar a la variedad de español local la versión de los textos y ejercicios en castellano. Este proceso fue revisado minuciosamente por una profesora universitaria experta en lingüística para garantizar la pertinencia y el uso correcto del lenguaje empleado. Además, se procuró que las imágenes y las referencias culturales utilizadas guardaran una mayor relación con la realidad de Lima urbana.

Con respecto a la adecuación tecnológica, esta se realizó teniendo en cuenta las limitaciones referidas al ancho de banda disponible para conexión de internet y la infraestructura instalada en los colegios de zonas urbanas de la ciudad. Asimismo, se tuvo especial consideración en garantizar la existencia de tres elementos importantes: (1) la optimización de audios e imágenes para lograr un mayor aprovechamiento de esos medios, (2) el uso de estándares de la W3C para facilitar el acceso a internet, y (3) el empleo de software libre para asegurar la posibilidad de compartir la aplicación con otras organizaciones que la requieran. Como parte del proceso de desarrollo de la plataforma LEO se probó su funcionamiento en una etapa inicial con una docente y ocho estudiantes de 
similares características y grado de los participantes. Esta prueba piloto confirmó que la funcionalidad de la plataforma era adecuada y que el diseño de la misma era atractivo. Se incorporaron algunas recomendaciones que permitieron lograr mayor precisión en las instrucciones, en los mensajes de retroalimentación y algunos ejercicios planteados.

Proceso de intervención. Se llevó a cabo en tres momentos: (1) el contacto y selección de los colegios participantes, (2) la capacitación a los docentes, y (3) la intervención propiamente dicha. Para el contacto con los participantes se realizó una convocatoria a los colegios que pertenecen a la red de instituciones educativas del Instituto de Informática de la Universidad - InfoPUC (70 aproximadamente). Se visitó a las instituciones interesadas y se evaluó el cumplimiento de los requisitos de inclusión. La capacitación de las docentes del grupo experimental consistió en dos sesiones de tres horas donde se presentaron los fundamentos teóricos de la propuesta, el manejo del entorno virtual y los lineamientos para la aplicación de la plataforma con los estudiantes. La intervención tuvo una duración de 12 semanas. Se trabajó en dos sesiones de una hora y media por semana, y se llevó a cabo en los laboratorios de computación de la institución participante. Cada sesión fue acompañada por dos miembros del equipo de investigación: uno encargado de solucionar posibles dificultades con la plataforma y otro responsable de registrar cómo se llevaba a cabo la sesión y de resolver las dudas pedagógicas.

Recolección de información. Antes de iniciar el uso de la plataforma, se aplicaron las pruebas de evaluación de la comprensión de lectura y de las palabras usadas en el programa. Al finalizar, se administraron nuevamente las mismas pruebas. Cada sesión en la que se empleó el programa se registró mediante fichas de observación. Además, con las docentes que aplicaron LEO se realizaron dos reuniones: una individual y una grupal, con el objetivo de recoger sus percepciones y opiniones sobre la experiencia, y proveerles de feedback sobre la aplicación. Por último, se realizó una reunión de cierre con las docentes y los directivos de los colegios seleccionados, así como una sesión final con los estudiantes que participaron en la experiencia. 


\section{Resultados}

Los hallazgos fueron organizados en tres aspectos: (1) los puntajes obtenidos en las pruebas aplicadas antes y después de la intervención, (2) el desempeño de los estudiantes en la plataforma, y (3) la experiencia del uso de LEO por parte de los docentes.

\section{Puntajes de las pruebas de entrada y salida}

Se correlacionaron los puntajes de comprensión para textos narrativos (CLN) e informativos (CLI) con los de vocabulario (V) de toda la muestra. En la evaluación de entrada se halló una correlación significativa y medianamente fuerte entre V y CLN $(p=.001 ; r=.34)$ y CLI $(p=.001 ; r=.34)$. En cuanto a la medida de V de salida, también se encontró mediana correlación con los puntajes finales de CLN $(p=.000 ; r=.48)$ y CLI $(p=.000 ; r=.42)$. Para comparar el rendimiento de los grupos experimental y control respecto a estas variables dependientes, se emplearon pruebas de contraste de medias y medianas. En la Tabla 1 se observan las medidas de tendencia central para toda la muestra según los puntajes de los dos instrumentos administrados. Según los resultados obtenidos, al finalizar la aplicación de la plataforma se observaron diferencias significativas entre los grupos en todas las medidas. Los promedios obtenidos señalan mayores puntajes en el grupo experimental, que empleó la plataforma, frente al grupo control que no la utilizó. 
Efecto de una plataforma virtual en comprensión de lectura y vocabulario / Thorne et al.

\section{Tabla 1}

Medidas de tendencia central de los puntajes de las pruebas de entrada y salida para ambos grupos

\begin{tabular}{|c|c|c|c|c|c|c|c|}
\hline \multirow{2}{*}{ Grupo } & \multirow{2}{*}{ Medida } & \multicolumn{3}{|c|}{ Prueba de entrada } & \multicolumn{3}{|c|}{ Prueba de salida } \\
\hline & & $M$ & $\mathrm{Me}$ & D.E. & $M$ & $M e$ & D.E. \\
\hline \multirow{2}{*}{ Experimental } & CLN & 10.80 & 11.00 & 2.45 & 11.71 & 12.00 & 1.88 \\
\hline & CLI & 13.47 & 14.00 & 2.59 & 13.67 & 14.00 & 2.69 \\
\hline$(n=49)$ & V & 18.82 & 19.00 & 3.38 & 21.55 & 22.00 & 2.71 \\
\hline \multirow[t]{2}{*}{ Control } & CLN & 10.41 & 11.00 & 2.92 & 10.67 & 11.00 & 2.12 \\
\hline & CLI & 11.54 & 12.00 & 4.10 & 12.00 & 13.00 & 3.85 \\
\hline$(n=39)$ & V & 17.72 & 18.00 & 3.19 & 18.26 & 19.00 & 4.71 \\
\hline
\end{tabular}

Asimismo, como se observa en la Tabla 2, la comparación de puntajes antes de empezar la implementación indica que no existían diferencias estadísticamente significativas entre los grupos en CLN y V. No obstante, sí se encontraron diferencias iniciales estadísticamente significativas en CLI, inclinándose a favor del grupo experimental.

\section{Tabla 2}

Pruebas t de student y $U$ de Mann-Whitney de los puntajes de las pruebas de entrada y salida para ambos grupos

\begin{tabular}{lcccccc}
\hline \multirow{2}{*}{ Estadístico } & \multicolumn{3}{c}{ Prueba de entrada } & \multicolumn{3}{c}{ Prueba de salida } \\
& CLN & CLI & V & CLN & CLI & V \\
\hline t de Student $(86 \mathrm{gl})$ & -.67 & - & -1.55 & - & - & - \\
U de Mann-Whitney & - & 714.00 & - & 646.00 & 733.00 & 506.00 \\
Sig. (bilateral) & .50 & $.04^{*}$ & .12 & - & - & - \\
Sig. (unilateral) & - & - & - & $.00^{* *}$ & $.03^{*}$ & $.00^{* * *}$ \\
\hline
\end{tabular}

${ }^{*} p<.05, " * p<.01, " * p<.001$

Seguidamente, se compararon estos puntajes al interior de cada grupo (ver Tabla 3). En el caso del grupo experimental, se hallaron 
diferencias estadísticamente significativas entre los puntajes iniciales y finales en CLN y V. Los promedios de estas medidas indican que los puntajes en ambas capacidades al final de la intervención resultaron mayores que los de entrada $\left(\mathrm{CLN} M_{\text {Expsalida }} 11.71\right.$ Vs. CLN $M_{\text {ExpEnt }} 10.80$; y VM $M_{\text {Expsalida }} 21.55$ Vs. VM $M_{\text {ExpEnt }} 18.82$ ). En el grupo control, se observa que solo existieron diferencias estadísticamente significativas entre las medidas de V. Los promedios obtenidos señalan que el puntaje final es superior respecto al inicial.

\section{Tabla 3}

Prueba de Wilcoxon de los puntajes de las pruebas de entrada y salida para ambos grupos

\begin{tabular}{llccc}
\hline \multirow{2}{*}{ Grupo } & \multirow{2}{*}{ Estadístico } & CLN salida - & CLI salida - & V salida \\
& & CLN entrada & CLI entrada & V entrada \\
\hline \multirow{2}{*}{ Experimental } & Z & -2.78 & -.29 & -4.70 \\
& Sig. (unilateral) & $.00^{* *}$ & .39 & $.00^{* * *}$ \\
\multirow{2}{*}{ Control } & Z & -.35 & -.67 & -1.73 \\
& Sig. (unilateral) & .36 & .25 & $.04^{*}$ \\
\hline
\end{tabular}

${ }^{*} p<.05,{ }^{* *} p<.01,{ }^{* * *} p<.001$

Por último, se calculó el tamaño del efecto de la intervención (ver Tabla 4). Respecto a las diferencias previas a la aplicación de la plataforma, se observa que solo fueron significativas en CLI con un tamaño mediano ( $\mathrm{d}_{\text {CLI Ent }}=.58$ ). Esto confirma la existencia de discrepancias de antemano entre los grupos en dicha medida. Al final de la intervención, las diferencias entre los grupos fueron significativas, con una magnitud mediana en CLN ( $\left.\mathrm{d}_{\text {CLN Sal }}=.52\right)$ y grande en $\mathrm{V}$ $\left(\mathrm{d}_{\text {vsal }}=.89\right)$. En cuanto a CLI, se aprecia que las diferencias iniciales prácticamente se han mantenido $\mathrm{y}$, por lo tanto, no existe un efecto significativo. 


\section{Tabla 4}

Tamaño del efecto (d de Cohen) de los puntajes de las pruebas de entrada y salida para ambos grupos

\begin{tabular}{lcccccc}
\hline \multirow{2}{*}{ Estadístico } & \multicolumn{3}{c}{ Prueba de entrada } & \multicolumn{3}{c}{ Prueba de salida } \\
& CLN & CLI & V & CLN & CLI & V \\
\hline d de Cohen & .14 & .58 & .33 & .52 & .51 & .89 \\
Sig. & .50 & $.04^{*}$ & .12 & $.00^{* *}$ & $.03^{*}$ & $.00^{* * *}$ \\
\hline
\end{tabular}

${ }^{*} p<.05,{ }^{* * *} p<.01, " * * * 0<001$

\section{Desempeño de los estudiantes en la plataforma}

Se identificaron algunos datos generales sobre el desarrollo del programa a lo largo de la intervención. En los dos primeros textos, los estudiantes necesitaron tres horas (equivalente a dos sesiones) para leer y resolver los ejercicios de cada texto, luego de lo cual varió el tiempo de acuerdo a las habilidades de cada estudiante. Ello coincide con que a partir del cuarto y quinto texto, las docentes permitieron que los estudiantes avancen sin esperar al resto del salón y con menores indicaciones de su parte. En cuanto al desarrollo de las actividades del programa, los estudiantes leyeron y completaron todas las actividades de siete cuentos en promedio. El porcentaje de alumnos que acabó todas las tareas de un texto disminuyó conforme el orden de las lecturas (que incrementan progresivamente su complejidad): 96\% terminó las dos primeras, 94\% la tercera, 88\% la cuarta, $86 \%$ las tres siguientes, y $67 \%$ la última. En relación a lo anterior, las actividades de vocabulario fueron terminadas por mayor porcentaje de estudiantes, seguidas de los ejercicios de comprensión de lectura (estrategias), y de las actividades de evaluación de la comprensión y del vocabulario (ver Figura 2). 


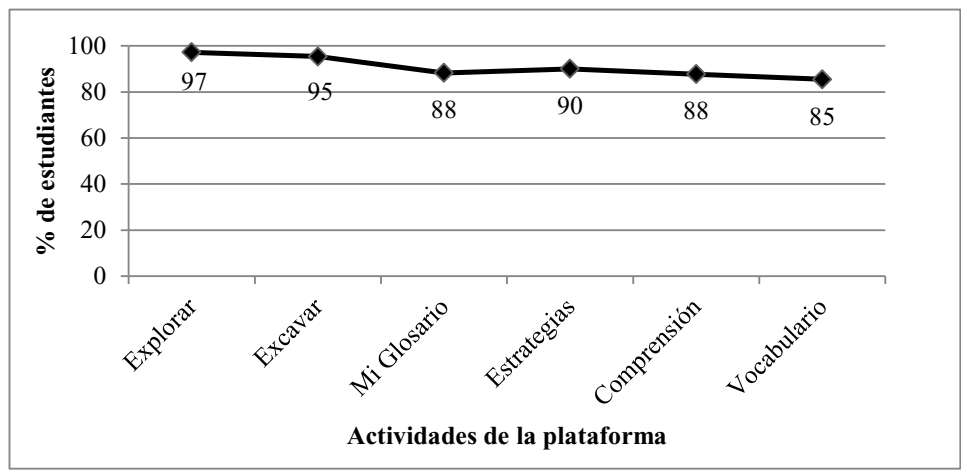

Figura 2. Porcentaje de alumnos que completaron cada actividad del programa

Por otro lado, se analizaron los puntajes de las evaluaciones de cada lectura insertas en la plataforma LEO, que fluctúan entre 0 y 20 puntos. Las medias obtenidas evidenciaron un mejor rendimiento en vocabulario que en comprensión, aunque ambas medidas disminuyeron conforme las lecturas se hicieron más complejas. Los puntajes en vocabulario muestran un descenso a partir del tercer texto, donde se mantuvieron entre 10 y 12 puntos hasta la última lectura. En comprensión, los puntajes decrecieron a partir del cuarto texto y se agudizó la disminución en los dos textos informativos siguientes (ver Figura 3). En ambas evaluaciones, los puntajes de los textos narrativos fueron mayores que los de los textos informativos desde la tercera lectura, pero esta diferencia fue más acentuada en comprensión.

Los puntajes de las evaluaciones de comprensión evidenciaron un mejor rendimiento en las preguntas literales que en las inferenciales, especialmente en dos lecturas: en la primera, que presentó $77 \%$ de acierto en las preguntas literales y $46 \%$ en las inferenciales; y en la quinta, que reportó $79 \%$ de respuestas correctas para las preguntas literales y $43 \%$ para las inferenciales (ver Figura 4). 


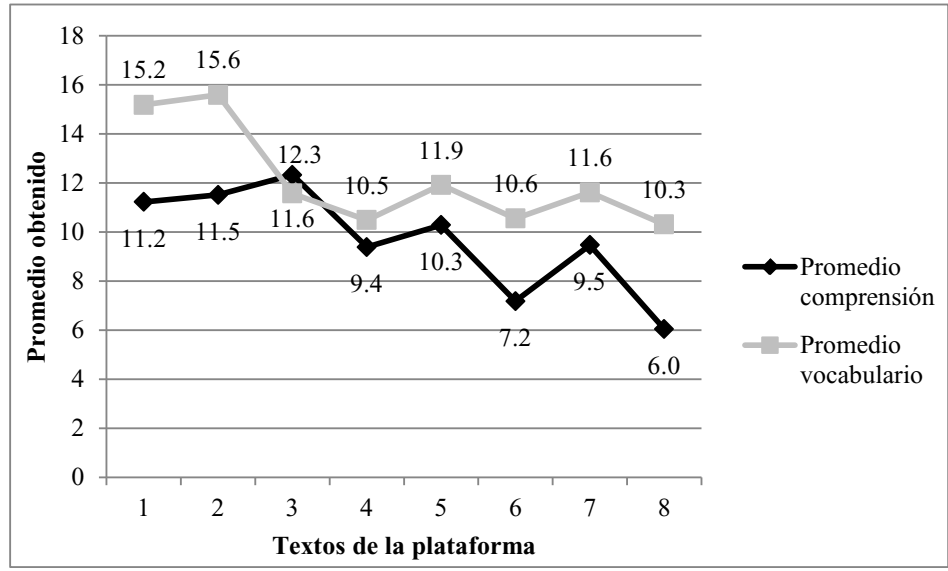

Figura 3. Promedio de los puntajes de las evaluaciones de comprensión de lectura y vocabulario para cada texto

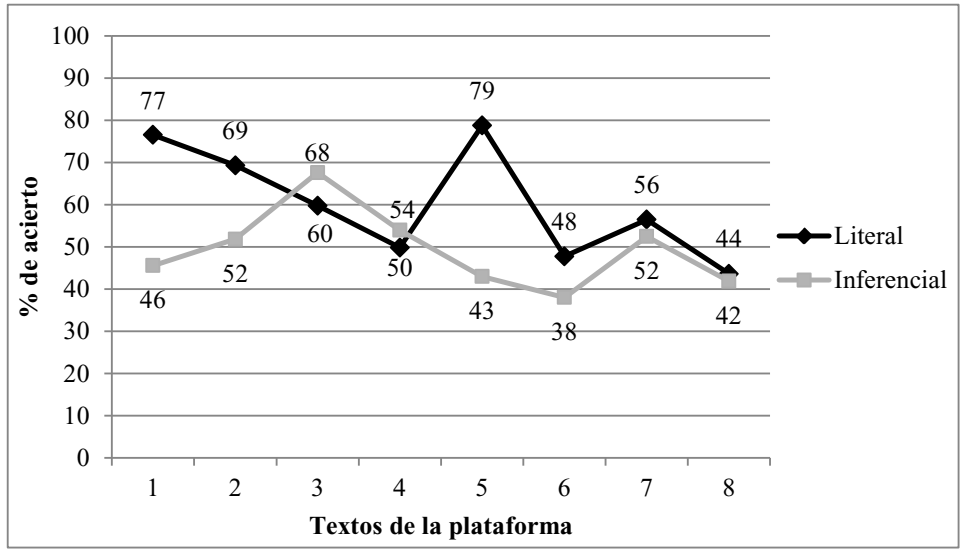

Figura 4. Porcentaje de acierto en la evaluación de comprensión de lectura para cada texto según tipo de pregunta

En cuanto a las evaluaciones de vocabulario, se obtuvo un mayor porcentaje de acierto en el nivel "Hábil", que presentó una disminución en el porcentaje de acierto en las lecturas cuatro y seis, pero que logró casi el 100\% de respuestas correctas en el texto final. El nivel 
"Sabio" aparece como segundo en porcentaje promedio de acierto, mostrando menores variaciones a lo largo de los textos. Por último, el nivel "Experto" mostró mayores disparidades entre las lecturas: hacia la tercera disminuyó alrededor de $40 \%$ y, a pesar de aumentar progresivamente, el porcentaje de acierto del texto final no logró superar el máximo obtenido en este nivel (ver Figura 5).

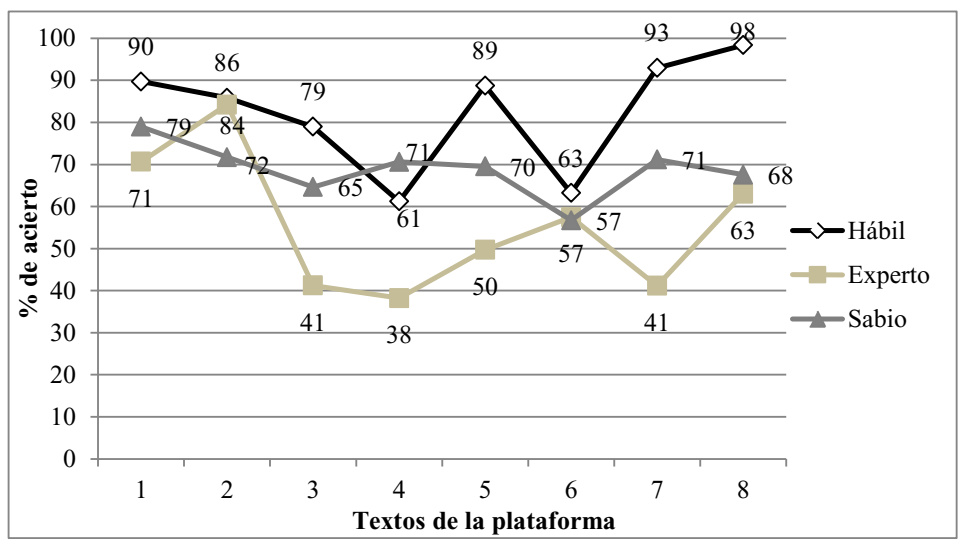

Figura 5. Porcentaje de acierto en la evaluación de vocabulario para cada texto según nivel de dificultad

\section{Experiencia de las docentes con el uso de LEO}

Se identificaron tres aspectos que sintetizan las percepciones y opiniones de las docentes respecto al empleo de la plataforma y su participación en el proyecto: (1) los factores positivos percibidos para los alumnos, (2) los logros y aprendizajes personales de las docentes, y (3) las dificultades enfrentadas a lo largo de la intervención. Ello se puede apreciar en la Tabla 5, en donde se incluye la información obtenida a través de las guías de observación de aula para complementar los datos sobre las dificultades técnicas. 
Efecto de una plataforma virtual en comprensión de lectura y vocabulario / Thorne et al.

\section{Tabla 5}

Principales categorias encontradas en la opinión de las docentes sobre el uso de LEO

\begin{tabular}{|c|c|c|c|}
\hline $\begin{array}{l}\text { Aspectos positivos } \\
\text { para los alumnos }\end{array}$ & Logros & $\begin{array}{l}\text { Dificultades } \\
\text { propias del } \\
\text { docente }\end{array}$ & $\begin{array}{l}\text { Dificultades } \\
\text { técnicas }\end{array}$ \\
\hline $\begin{array}{l}\text { Promueve la } \\
\text { motivación y } \\
\text { atención de los } \\
\text { estudiantes. }\end{array}$ & $\begin{array}{l}\text { Vencer sus } \\
\text { temores frente } \\
\text { al uso de la } \\
\text { tecnología. }\end{array}$ & $\begin{array}{l}\text { Poco conocimiento } \\
\text { en informática y } \\
\text { computación. }\end{array}$ & $\begin{array}{l}\text { Algunas veces } \\
\text { fallaban los } \\
\text { audios del texto. }\end{array}$ \\
\hline \multirow[t]{2}{*}{$\begin{array}{l}\text { El tratamiento de } \\
\text { los contenidos ha } \\
\text { ayudado a mejorar } \\
\text { la expresión } \\
\text { escrita. }\end{array}$} & $\begin{array}{l}\text { Incorporación } \\
\text { de elementos de } \\
\text { la plataforma } \\
\text { en sus clases y } \\
\text { evaluaciones. }\end{array}$ & $\begin{array}{l}\text { Falta de mayor } \\
\text { exploración previa } \\
\text { de la plataforma, } \\
\text { sobre todo desde el } \\
\text { perfil docente. }\end{array}$ & $\begin{array}{l}\text { Fallas diversas } \\
\text { con los agentes } \\
\text { interactivos. }\end{array}$ \\
\hline & $\begin{array}{l}\text { Alto interés de } \\
\text { replicar el uso de } \\
\text { la plataforma en } \\
\text { el } 2011 \text { en 4to, } \\
5 \text { to y 6to grados. }\end{array}$ & $\begin{array}{l}\text { Enviar los } \\
\text { mensajes de retro- } \\
\text { información. }\end{array}$ & $\begin{array}{l}\text { No contar con } \\
\text { los textos con } \\
\text { anticipación. }\end{array}$ \\
\hline
\end{tabular}

En general, la opinión de las docentes en relación al uso del programa fue positiva. Reconocieron que la plataforma no solo generó resultados beneficiosos en cuanto al aprendizaje de los estudiantes, sino también para su propio desarrollo profesional como maestras. La principal barrera que consideran haber enfrentado fue el uso de la tecnología. Sin embargo, reportaron haber incorporado elementos del entorno virtual como herramienta para el aprendizaje de la comprensión de lectura. Es importante mencionar que las docentes reconocieron que el acompańamiento constante brindado a lo largo de la implementación del programa fue fundamental para alcanzar los logros expuestos. En la reunión de cierre, tanto las directoras de los colegios como las docentes manifestaron que muchas iniciativas hacen capacitación, pero suelen realizar visitas solo a la mitad y al final del proyecto. Por ello, rescataron el acompañamiento técnico y pedagógico constante como una fortaleza del programa LEO. 


\section{Discusión}

Los resultados presentados permiten reconocer la efectividad de la plataforma LEO como herramienta para mejorar la comprensión de lectura de textos narrativos y el vocabulario en estudiantes de quinto grado de primaria de escuelas de nivel socioeconómico medio-bajo de Lima. Sin embargo, en esta misma población no se observaron avances significativos en la comprensión de textos informativos. Estos resultados fueron consistentes tanto en las pruebas de salida como en las actividades de evaluación de la plataforma. Con respecto a los resultados en comprensión de textos narrativos, dichos hallazgos sugieren que las estrategias desarrolladas en LEO son pertinentes para optimizar las capacidades de comprensión de este tipo de lecturas. Asimismo, otro aspecto que puede haber contribuido a estos resultados es la participación anual de las instituciones educativas en el Plan Lector ${ }^{1}$, el cual privilegia la lectura y la evaluación de obras literarias de carácter narrativo, favoreciendo el aprendizaje previo respecto a textos de este tipo.

Sin embargo, el uso de las estrategias utilizadas en LEO no ha dado los mismos resultados para los textos informativos. Si bien diferentes autores (Dalton \& Proctor, 2007; Pressley, 2002; Strickland, 2002; Williams, 2007) señalan que dichas estrategias mejoran la comprensión lectora, en el presente estudio se ha evidenciado que su efecto no es tal para textos de carácter no narrativo. En la medida que la comprensión de lectura es un proceso dinámico entre el texto, el lector y la actividad, supone un ajuste de las estrategias y habilidades del lector para lograr el éxito en cada tarea particular, tal como lo señalan Snow y Sweet (2003). Estos resultados ponen en evidencia la necesidad - fundamentada por la investigación reciente - de diseñar estrategias de comprensión que respondan específicamente a las características de los textos informativos.

1 El Plan Lector fue implementado por el Ministerio de Educación en 2006. Propone la lectura de 12 textos para cada grado a lo largo del año escolar, con el propósito de incrementar el hábito de leer y desarrollar capacidades de comprensión de lectura en estudiantes y docentes. 
Un punto de referencia útil es propuesto por Braslavsky (2005), quien diferencia entre textos informativos y expositivos: los primeros corresponden a periódicos (entrevistas y noticias) y publicidad (folletos y afiches), mientras que los segundos presentan datos, hechos, investigaciones, es decir, exponen un tema con el fin de generar nuevos conocimientos. De acuerdo a esta clasificación, LEO estaría empleando solo textos de tipo expositivo, para cuya lectura se identifican estrategias especialmente necesarias, como activar los conocimientos previos del estudiante y responder preguntas de comprensión a lo largo del texto (Dymock \& Nicholson, 2010). De igual manera, se reconoce que el empleo de textos solo expositivos en LEO no ha facilitado que los estudiantes transfieran las habilidades adquiridas hacia otros tipos de textos informativos como los mencionados. Así, los resultados de las evaluaciones de entrada y salida administradas a los participantes del estudio, que emplean una lectura informativa de tipo publicitario, pueden haberse visto afectadas por la poca exposición de los estudiantes a ese tipo de textos y la poca ejercitación en estrategias acordes a los mismos.

Por lo tanto, es posible imaginar que la plataforma LEO pudiera haber en efecto mejorado las destrezas de comprensión de textos expositivos, pero que la prueba de salida no fuera la evaluación más apropiada para captar esa mejoría, debido a que los textos informativos evaluados eran principalmente de tipo publicitario. Si bien este estudio no ofrece información suficiente para poder confirmar esta opción, los resultados señalan dos necesidades para el trabajo futuro con la plataforma: (1) ampliar la variedad de tipos de textos informativos incluidos en el entorno virtual, y (2) diseñar estrategias que respondan específicamente a las características de dicha clase de textos. En lo concerniente a la comprensión de los aspectos literales e inferenciales del texto, se aprecia que el desempeño de los estudiantes en LEO confirma lo hallado en las últimas evaluaciones nacionales (Ministerio de Educación, 2008a, 2010a, 2011, 2012): existe un menor porcentaje de acierto en las preguntas de tipo inferencial, indicador que se hace más severo en los textos informativos. 
Respecto a la adquisición de vocabulario, los resultados muestran mayores ganancias en esta capacidad para los estudiantes del grupo experimental: los estadísticos son más contundentes e indican que la tendencia a favor de este grupo se incrementa luego de la intervención. Estos hallazgos muestran la efectividad de LEO para desarrollar el vocabulario, pues los alumnos que emplearon la plataforma aprendieron el significado y uso de las palabras a las que fueron expuestos. Asimismo, los resultados favorecen la relación positiva entre el vocabulario y la comprensión de textos tanto narrativos como informativos, lo cual apoya la evidencia ya encontrada por otros autores (Morales, 2009; Nagy, 1998 citados en Dalton \& Proctor, 2007). En esta línea, la literatura especializada reporta que un inadecuado bagaje de vocabulario se relaciona con dificultades en la comprensión de lectura (Lipson \& Wixson, 1997; Paris, Wasik \& Turner, 1991 citados en Dalton \& Proctor, 2007). De acuerdo a ello, se podría recomendar utilizar las actividades empleadas en LEO para la ampliación del vocabulario. Por ejemplo, la identificación del significado de las palabras más importantes o difíciles de un texto antes de leerlo ayudaría a que la lectura sea más fluida y se recojan aprendizajes previos importantes para la comprensión. Además, reforzar el significado y uso de este vocabulario luego de la lectura consolidaría el aprendizaje de este bagaje léxico, que ejerce gran influencia en la comprensión lectora.

Otro aspecto central que sugieren los resultados del estudio es la relevancia del rol del docente en el uso efectivo de la plataforma. Los datos obtenidos indican un adecuado desarrollo del programa, pero la necesidad de ampliar el tiempo destinado a su empleo (en vez de 12, 13 o 14 semanas) para completarlo. Además, se advierte que la facilitación del docente en este proceso ha sido de especial relevancia, no solo porque los estudiantes mostraron poca autonomía y autorregulación para utilizar la plataforma, sino especialmente porque el maestro cumple una función central al aclarar, discutir y elaborar con el estudiante aspectos de su trabajo en el entorno virtual. Los puntajes obtenidos en el primer y segundo texto evidencian de alguna manera que el acompańamiento cercano mejora el desempeño de los estudiantes en 
las tareas propuestas. Estos resultados resaltan la necesidad de que el docente asuma un rol activo como agente que potencia la capacidad de la plataforma como mediadora de la comprensión lectora. Por ello, la capacitación y el acompañamiento han sido fundamentales para fortalecer el rol de las docentes (Chesterfield et al., 2005; Morales, 2009; Sunkel, 2006; Villalón et al., 2005; Villatoro \& Silva, 2005). El acompańamiento pedagógico y tecnológico ofrecido a las profesoras ha sido reconocido por ellas como un factor clave para lograr vencer su temor hacia la tecnología, manejar las funciones básicas de LEO e incorporar algunos elementos de la plataforma en sus clases.

Este estudio presenta resultados satisfactorios que contribuyen a formular y explorar soluciones eficientes para atender uno de los problemas centrales de la educación en el país: el bajo nivel de comprensión de lectura de nuestros estudiantes. Si bien la plataforma LEO se encuentra aún lejos de ofrecer una solución comprehensiva o viable para la diversidad de instituciones educativas del país, es una herramienta que emerge con un gran potencial como instrumento de investigación, mediador en la capacitación docente, y entorno capaz de motivar y elevar la comprensión de lectura en escuelas urbanas de nivel socioeconómico medio-bajo. Los resultados de este estudio son positivos, pero presentan también algunas limitaciones. Por un lado, solo se ha evaluado el programa en una muestra restringida, por lo que el potencial de la plataforma para atender a poblaciones más diversas aún requiere de investigación futura. Por ejemplo, queda pendiente explorar la potencialidad de LEO en nuevos contextos, diferentes grados de escolaridad, y emplear evaluaciones estandarizadas para el caso de vocabulario, que den cuenta de efectos no solo en el aprendizaje de palabras del entorno, sino en el bagaje léxico de los estudiantes. Por otro lado, se considera necesario mejorar el servicio ofrecido por el programa. Específicamente, sería recomendable ajustar el diseño del módulo para el docente y reforzar su ejercitación, de forma que este domine su uso antes de la intervención y mejore el envío de retroalimentación por medio del entorno virtual. Además, sobre la plataforma, se reconoce la necesidad de optimizar los audios y agentes para agilizar su funcionamiento. 
Finalmente, es posible afirmar que este estudio ofrece solo un primer avance en un tema que requiere mayor investigación, pero muestra ya evidencia prometedora de avances significativos en comprensión de lectura como resultado de la intervención educativa implementada. Se espera que este ejemplo de investigación aplicada motive a más investigadores a trabajar con cercana colaboración con maestros, directores de escuela y alumnos para juntos avanzar gradualmente hacia soluciones creativas, acertadas y viables para la realidad de nuestras escuelas.

\section{Referencias}

Alvermann, D. E. \& Eakle, A. J. (2003). Comprehension instruction: adolescents and their multiple literacies. En A. P. Sweet \& C. Snow. (Eds.). Rethinking reading comprehension. New York: The Guilford Press.

Braslavsky, B. (2005). Enseñar a entender lo que se lee. La alfabetización en la familia y la escuela. Buenos Aires: Fondo de Cultura Económica de Argentina.

Bravo, L., Villalón, M. \& Orellano, E. (2005). El rendimiento en la lectura en el Primer Ciclo Básico y algunos procesos psicolingüísticos de ingreso. Boletín de Investigación Educacional, 20(1), 51-63.

Carreño, B. (2000). Comprensión de lectura al finalizar primaria en niños peruanos. Tesis de Maestría no publicada. Facultad de Letras y Ciencias Humanas, Especialidad de Psicología. Lima: Pontificia Universidad Católica del Perú.

Cazden, C. B. \& Beck, S. (2005). Classroom discourse. En A. C. Graesser, M. Gernsbacher \& S. R. Goldman. (Eds.). Handbook of discourse processes. New Jersey: Lawrence Erlbaum Associates.

Chall, J. (1996). Stages of reading development. Florida: Harcourt Brace College Publishers.

Chesterfield, R., Culver, K., Hunt, B. C. \& Linan-Thompson, S. (2005). Un estudio reflexivo del desarrollo profesional de los docentes 
Efecto de una plataforma virtual en comprensión de lectura y vocabulario / Thorne et al.

en los centros regionales de América Latina y el Caribe para la excelencia de la capacitación a docentes. California: USAID - Aguirre International.

Claux, M. L. \& La Rosa, M. I. (2004). La comprensión de lectura en el aula: una experiencia significativa. Lima: Centro para el Magisterio Universitario PUCP.

Dalton, B. \& Proctor, P. (2007). Reading as thinking: integrating strategy instruction in a universally designed digital literacy environment. En D. S. McNamara. (Ed.). Reading comprehension strategies: theories, interventions and technologies. New Jersey: Lawrence Erlbaum Associates.

Dalton, B., Proctor, P., Uccelli, P., Mo, E. \& Snow, C. (2011). Designing for diversity: the role of reading strategies and interactive vocabulary in a digital reading environment for fifth-grade monolingual English and bilingual students. Journal of Literacy Research, 43(1), 68-100.

Dymock, S. \& Nicholson, T. (2010). "High 5!" Strategies to enhance comprehension of expository text. The Reading Teacher, 64(3), 166-178.

Gómez-Palacio, M., Gonzáles, L., López-Arraiza, M. L. \& Adame, M. G. (1993). Indicadores de la comprensión lectora. Washington, D. C.: OEA.

Guthrie, J. T., Wigfield, A. \& Prencevich, K. C. (2004). Motivating reading comprehension: concept-oriented reading instruction. New Jersey: Lawrence Erlbaum Associates.

Henao, O. (2006). Evidencias de la investigación sobre el impacto de las tecnologías de información y comunicación en la enseńanza de la lecto-escritura. Revista Educación y Pedagogía, 18(44), 73-87.

Henao, O. \& Ramírez, D. (2007). Estrategias de exploración y construcción de significado utilizadas por estudiantes de educación básica durante la lectura de textos en formato hipermedial. INNOVAR. Revista de Ciencias Administrativas y Sociales, 17(30), 47-57. 
Kintsch, W. \& Kintsch E. (2005). Comprehension. En S. G. Paris \& S. A. Stahl. (Eds.). Children's reading comprenhension and assessment. New Jersey: Lawrence Erlbaum Associates.

Ministerio de Educación del Perú. Unidad de Medición de la Calidad Educativa. (2001). Boletin UMC No 9. El Perú en el primer estudio internacional comparativo de la UNESCO sobre lenguaje, matemática y factores asociados en tercer y cuarto grado. Recuperado de http://www2.minedu.gob.pe/umc/admin/images/ publicaciones/boletines/Boletin-09.pdf

Ministerio de Educación del Perú. Unidad de Medición de la Calidad Educativa. (2005). Evaluación Nacional del Rendimiento Estudiantil 2004. Recuperado de http://www2.minedu.gob.pe/ umc/admin/images/en2004/MatematicaS3_5.pdf

Ministerio de Educación del Perú. Unidad de Medición de la Calidad Educativa. (2008a). Resultados de la Evaluación Censal de Estudiantes 2007. Recuperado de http://www2.minedu.gob.pe/ umc/presentaciones_index.php

Ministerio de Educación del Perú. Unidad de Medición de la Calidad Educativa. (2008b). Resultados del Segundo Estudio Regional Comparativo y Explicativo. Recuperado de http:// www2.minedu.gob.pe/umc/index2.php? __codigo $=2018 v_{-}$ plantilla $=\mathrm{R}$

Ministerio de Educación del Perú. Unidad de Medición de la Calidad Educativa. (2010a). Resultados de la Evaluación Censal de Estudiantes 2009. Recuperado de http://www2.minedu.gob.pe/ umc/index2.php? v_codigo $=234 \& v \_$plantilla $=R$

Ministerio de Educación del Perú. Unidad de Medición de la Calidad Educativa. (2010b). Resultados de la Evaluación PISA 2009. Recuperado de http://www2.minedu.gob.pe/umc/index2. php?v_codigo $=193 \&$ v_plantilla $=R$

Ministerio de Educación del Perú. Unidad de Medición de la Calidad Educativa. (2011). Resultados de la Evaluación Censal de Estudiantes 2010. Recuperado de http://www2.minedu.gob.pe/ umc/index 2 .php? v_codigo $=246 \&$ v_plantilla $=\mathrm{R}$ 
Ministerio de Educación del Perú. Unidad de Medición de la Calidad Educativa. (2012). Resultados de la Evaluación Censal de Estudiantes 2011. Recuperado de http://www2.minedu.gob.pe/ umc/index $2 . p h p ?$ v_codigo $=2538 v$ _plantilla $=\mathrm{R}$

Moje, E. \& Hinchman, K. (2004). Culturally responsive practices for youth literacy learning. En T. Jetton \& A. Dole. (Eds.). Adolescent literacy research and practice. New York: The Guilford Press. Morales, S. (1999). Comprensión de lectura en alumnos de cuarto y quinto grado de primaria de Lima. Revista de Psicología, 17, 263-282.

Morales, S. (2009). Socio-cultural variables and reading literacy acquisition in Lima, Perú. Nijmegen: Radboud Universiteit Nijmegen.

Nakano, T. (1996). El programa curricular de articulación de inicial primer grado de primaria y el rendimiento en comprensión de lectura inicial en niños de primer grado. Tesis de Licenciatura. Lima: PUCP.

Pedró, F. (2011). Tecnología y escuela: lo que funciona y por qué. XXVI Semana Monográfica de la Educación. La Educación en la Sociedad Digital. Madrid: Fundación Santillana.

Pérez, M. J. (2005). Evaluación de la comprensión lectora: dificultades y limitaciones. Revista de Educación, núm. extraordinario, 121-138.

Perfetti, C. A., Landi, N. \& Oakhill, J. (2005). The acquisition of reading comprehension skills. En M. J. Snowling \& C. Hulme. (Eds.). The science of reading: a handbook. Massachussets: Blackwell Publishing.

Pinzás, J. (1993). Cognitive monitoring in reading comprehension. A study of differences among schools in Lima. Nijmegen: Radboud Universiteit Nijmegen.

Pizarro, R., Muñoz, M., Colarte, P., Hernández, C. \& Olmos, J. (2005). Automaticidad en la lectura, mastery learning y currículum del hogar: sistemas instruccionales efectivos en la promoción de altos rendimientos académicos en lenguaje y matemática, primeros básicos. Boletín de Investigación Educacional, 20(1), 65-94.

Ponce, H., López, M., Labra, J., Brugerolles, J. P. \& Tirado, C. (2007). Evaluación experimental de un programa virtual de 
entrenamiento en lectura significativa (e-PELS). Revista Electrónica de Investigación Psicoeducativa, 5(2), 399-432.

Pressley, M. (2002). Metacognition and self-regulated comprehension. En A. E. Farstrup \& S. J. Samuels. (Eds.). What research has to say about reading instruction. Newark: International Reading Association.

Proctor, P., Dalton, B., Uccelli, P. \& Snow, C. (2008). ICON: Goal 2 development award. Presentado en IES Principal Investigators' Meeting, Washington, D. C. Recuperado de http://www.cast. org/research/projects/icon.html

Proctor, P., Uccelli, P., Dalton, B. \& Snow, C. (2009). Understanding depth of vocabulary and improving comprehension online with bilingual and monolingual children. Reading and Writing, 25, 311-333.

Selman, R. L. (2003). The promotion of social awareness: powerful lessons from the partnership of developmental theory and classroom practice. New York: Russell Sage.

Snow, C. (2002). Reading for understanding. Toward an R\&D program in reading comprehension. California: RAND.

Snow, C. \& Sweet, A. P. (2003). Reading for comprehension. En A. P. Sweet \& C. Snow. (Eds.). Rethinking reading comprehension. New York: The Guilford Press.

Snow, C. \& Uccelli, P. (2009). The challenge of academic language. En D. R. Olson \& N. Torrance. (Eds.). The Cambridge handbook of literacy. Cambridge: Cambridge University Press.

Stahl, S. A. \& Nagy, W. E. (2006). Teaching word meanings. New Jersey: Lawrence Erlbaum Associates.

Strickland, D. S. (2002). The importance of effective early intervention. En A. E. Farstrup \& S. J. Samuels. (Eds.). What research has to say about reading instruction. Newark: International Reading Association.

Sunkel, G. (2006). Las nuevas tecnologias de la información y la comunicación (TIC) en la educación en América Latina. Una exploración 
de indicadores sociales. Santiago de Chile: CEPAL - Serie Políticas Sociales.

Thorne, C. (1991). A study of beginning reading in Lima. Nijmegen: Radboud Universiteit Nijmegen.

Thorne, C. \& Nakano, T. (2001). El rol del maestro en el aprendizaje de la lectura en el Perú. En A. Bazán. Enseñanza y evaluación de la lectura y escritura: algunos aportes de la investigación y la tecnología. Sonora: Instituto Tecnológico de Sonora.

Thorne, C. \& Pinzás, J. (1988). Factors affecting reading achievement in Peru. Presentado en Annual Meeting of the American Educational Research Association, New Orleans.

Trinidad, R. (2005). Entre la ilusión y la realidad. Las nuevas tecnologías en dos proyectos educativos del Estado. Lima: Instituto de Estudios Peruanos.

Verhoeven, L. \& Perfetti, C. A. (2011). Introduction to this special issue: vocabulary growth and reading skill. Scientific Studies of Reading, 15, 1-7.

Villalón, M., Silva, M., Razmilic, T. \& Swartz, S. L. (2005). AILEM Programme: a long-term intervention to promote literacy learning in low-performing primary schools in Chile. Early Years, 25(2), 97-111.

Villatoro, P. \& Silva, A. (2005). Estrategias, programas y experiencias de superación de la brecha digital y universalización del acceso a las nuevas tecnologias de información y comunicación (TIC). Un panorama regional. Santiago de Chile: CEPAL - Serie Políticas Sociales.

Whitehurst, G. J. \& Lonigan, C. J. (1998). Child development and emergent literacy. Child Development, 69(3), 848-872.

Williams, D. S. (2007). Literacy in the curriculum: integrating text structure and content area instruction. En D. S. McNamara. (Ed.). Reading comprehension strategies: theories, interventions and technologies. New Jersey: Lawrence Erlbaum Associates.

Recibido el 10 de octubre, 2012

Aceptado el 02 de enero, 2013 